\title{
Editorial:
}

\section{Retos de las instituciones de educación superior para su articulación en la Industria 4.0}

\author{
Challenges for the Articulation of Higher Education Institutions in Industry 4.0
}

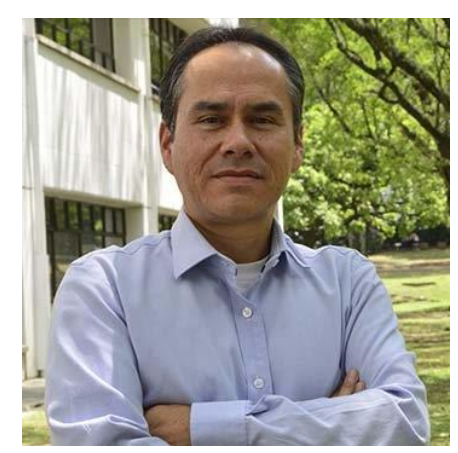

Juan Guillermo Pérez-Rojas

Magíster en Desarrollo

Rector

Instituto Tecnológico Metropolitano (ITM)

E-mail: juanperez@itm.edu.co

https://doi.org/10.22430/24223182.1584

La Cuarta Revolución Industrial (4RI), también conocida como Industria 4.0, hace referencia a un nuevo paradigma en el cual se le proporciona a la industria tradicional, y a sus procesos asociados, tecnologías donde los sistemas de información y comunicación se utilizan, en gran medida, para mejorar la productividad. Convergen entonces las tecnologías digitales, físicas y biológicas, por medio de la inteligencia artificial, el internet de las cosas, la biotecnología y los nuevos modelos de negocio (Schuh et al., 2014). La Industria 4.0 es entonces la base para la transición a una economía que se fundamenta en el conocimiento, dejando a un lado la economía de bajo costo y basada en los recursos, influyendo directamente en dimensiones como la educación y la formación de las nuevas generaciones. Esto plantea problemas de inmediata atención, especialmente en la educación postsecundaria, pues es una fase donde el individuo se capacita para ingresar a la vida laboral con pertinencia, por lo que las instituciones de educación superior -IES- necesitan enfocar sus esfuerzos en brindar todas las habilidades necesarias a los estudiantes, no solo para el presente sino también para enfrentar el trabajo del futuro (Gazzola \& Didriksson, 2018).

En este contexto, las IES insertas en las sociedades del conocimiento están llamadas a reinventarse, teniendo en cuenta los desafíos que la 4RI integra a las dinámicas formativas y sociales, donde 
convergen los desarrollos de la tecnología, la física y la biología, pues de seguir con el modelo tradicional, no estarán habilitadas para poder responder a las necesidades y retos a los que se enfrentan en un mundo que cada vez es más dinámico. Esta reinvención se debe centrar, tanto en la capacidad de desarrollo científico, como de innovación tecnológica, con el propósito de crecer y ser partícipe de la solución de los retos presentes en los nuevos campos del conocimiento y del trabajo. Por esto, las IES deben fomentar la innovación, tanto en la enseñanza como en el aprendizaje, basándose en la investigación científica, y promover nuevos métodos, tecnologías de la información y del aprendizaje, así como formas de organización.

En este sentido, Pedroza (2018) plantea que la tendencia educativa en las universidades que se enfrentan a la Industria 4.0 apunta hacia el concepto de una universidad innovadora que tiene en la investigación su eje principal de desarrollo. Este enfoque investigativo cubre dos planos relevantes: por un lado, la investigación se usa para la innovación científica y tecnológica aportando así nuevos conocimientos; por el otro, la investigación es usada en la innovación académica ayudando a redefinir las prácticas de aprendizaje y enseñanza. Lo cual da como resultado un modelo de universidad caracterizado por un dinamismo de retroalimentación entre estos dos planos.

Por la tanto, las IES deben enfocarse, no solo en formar a todos los miembros de su comunidad en los conocimientos básicos de las disciplinas o del trabajo, sino también en incentivar el desarrollo de habilidades y competencias múltiples, propias de las necesidades del contexto tecnológico, de forma que, desde las habilidades blandas como el trabajo colaborativo, la creatividad y el pensamiento crítico, se preparen egresados con una vocación hacia la innovación y la gestión tecnológica, desde las diversas áreas del conocimiento, pero con un fuerte apoyo en herramientas digitales y analíticas soportadas en el procesamiento de grandes volúmenes de información y desarrollo tecnológico.

Las IES deben ejercer un énfasis especial en la educación continua con la que puedan llegar a un gran número de profesionales, evitando que pierdan vigencia y se puedan insertar de manera exitosa, adquiriendo las habilidades necesarias para las dinámicas productivas que la Industria 4.0 está generando. Si queremos garantizar que los futuros profesionales estén pertinentemente preparados es necesario transmitir una comprensión de los conceptos en contexto, tal como aparecen en el mundo real. Las IES deben apostar por nuevos escenarios de formación y de práctica, en los cuales se pueda profundizar en estos otros conocimientos y habilidades, sin descuidar las competencias básicas que las estructuran, como las prácticas pedagógicas en áreas como matemáticas, comprensión lectora y ciencias, que giran en torno a mejorar la calidad de la educación. En dichos escenarios, los estudiantes podrán adquirir nuevas competencias aplicables a su profesión e integrar las habilidades blandas, trabajando de manera interdisciplinar y en equipo, promoviendo nuevas soluciones a los retos de la sociedad e incentivando el emprendimiento.

El Instituto Tecnológico Metropolitano con su nuevo Plan de desarrollo "A otro nivel" vincula las formas de relación que propone la 4RI a través de la revolución del talento, las habilidades centradas en el conocimiento, la innovación tecnológica y social, y la economía naranja; lo cual se combina para la generación de competencias propias de la nueva época y en la cual se contribuya a los ámbitos local, nacional e internacional, construyendo una nueva vocación tecnológica como un sello de identidad en el que se consolide la institución como un referente para la educación superior. 


\section{REFERENCIAS}

Gazzola, A. L., \& Didriksson, A. (2018). Tendencias de la educación superior en América Latina y el Caribe. URL

Pedroza Flores, R. (2018) La universidad 4.0 con currículo inteligente 1.0 en la cuarta revolución industrial. Revista Iberoamericana para la investigación y el desarrollo educativo RIDE. 9(17). 168194. https://doi.org/10.23913/ride.v9i17.377

Schuh, G., Potente, T., Wesch-Potente, C., Weber, A. R., \& Prote, J. P. (2014). Collaboration Mechanisms to Increase Productivity in the Context of Industrie 4.0. Procedia CIRP, 19, 51-56. https://doi.org/10.1016/i.procir.2014.05.016 